\author{
Journal Club
}

Editor's Note: These short, critical reviews of recent papers in the Journal, written exclusively by graduate students or postdoctoral fellows, are intended to summarize the important findings of the paper and provide additional insight and commentary. For more information on the format and purpose of the Journal Club, please see http://www.jneurosci.org/misc/ifa_features.shtml.

\title{
Corpus Callosum and Bimanual Coordination in Multiple Sclerosis
}

\author{
Brett W. Fling, ${ }^{1}$ Jessica A. Bernard, ${ }^{2}$ Jin Bo, ${ }^{1}$ and Jeanne Langan ${ }^{1}$ \\ ${ }^{1}$ Division of Kinesiology and ${ }^{2}$ Department of Psychology, University of Michigan, Ann Arbor, Michigan 48109 \\ Review of Bonzano et al. (http://www.jneurosci.org/cgi/content/full/28/12/3227)
}

Bimanual actions are ubiquitous in daily life. Many coordinated movements of the upper extremities, and in particular the hands, rely on precise timing of movements and interhemispheric communication via the corpus callosum. However, not all bimanual coordination tasks involve the corpus callosum. Other brain regions that typically contribute to bimanual movements include the cerebellum, supplementary motor area, cingulate motor cortex and premotor cortex with the level of recruitment dependent on the task, environment and amount of practice (cf. Swinnen and Wenderoth, 2004). Therefore, task selection is of great importance when investigating the contributions of different brain regions to performance.

The corpus callosum is the primary means of communication between hemispheres and plays a role in temporal coupling during continuous bimanual movements (Kennerley et al., 2002). This large, white-matter tract is a good candidate for exploration via diffusion tensor imaging (DTI). DTI, which assesses water diffusion, is a relatively new technique gaining popularity in research and clinical settings. Parallel fibers such as those in white matter impact the diffusion of water mol-

Received June 11, 2008; revised June 4, 2008; accepted June 5, 2008. We thank Dr. Rachael Seidler for critical reading of this manuscript. Correspondence should be addressed to Brett W. Fling, Neuromotor Behavior Laboratory, University of Michigan, 401 Washtenaw Avenue, Ann Arbor, Ml 48109-2214. E-mail: bfling@umich.edu.

D01:10.1523/JNEUROSCI.2157-08.2008

Copyright $\odot$ 2008 Society for Neuroscience $\quad$ 0270-6474/08/287248-02\$15.00/0 ecules, with higher rates of diffusion occurring parallel to the fibers. Fractional anisotropy (FA) is often used to quantify DTI data. FA is an index that ranges from 0 to 1 , with 1 indicating highly directional diffusivity. Diffusion data may also be used in tractography to illustrate the structure of white matter tracts (cf. Nucifora et al., 2007).

In cases where the integrity of white matter is disrupted, such as multiple sclerosis (MS), DTI has been shown to be an effective tool in identifying brain anomalies. Individuals with MS have reduced $\mathrm{FA}$, indicative of microstructural tissue damage (Lin et al., 2007). However, the relationship between this tissue damage and functional performance is not well understood.

In a recent study published in The Journal of Neuroscience Bonzano et al. (2008) used DTI to study the relationship between corpus callosum integrity and functional bimanual performance in patients with MS. Participants performed a bimanual repetitive finger opposition movement of thumb to index, medium, ring, and little fingers with both hands simultaneously. Movements were paced with an auditory stimulus at three randomly presented frequencies $(1,1.5$, and 2 $\mathrm{Hz}$ ). Time of the auditory cue and each finger touch was recorded through sensor-engineered gloves worn on both hands. Bimanual motor impairments were assessed via the interhand interval (IHI), the absolute value of the timing difference between movements of equivalent fingers of the two hands. Using this definition, the larger the IHI absolute value, the more severe the impairment. This measure was further separated into $\mathrm{IHI}_{\text {onset }}$, the absolute time difference between touch onset occurring in the left hand and the matching touch of the right hand, and $\mathrm{IHI}_{\text {offset }}$, the absolute time difference between the finger touch offset in the two hands. The authors also investigated the presence of possible impairments in finger movement sequences for MS patients by quantifying touch duration and intertapping interval. Similar to previous work (cf. Nucifora et al., 2007), Bonzano et al. (2008) used DTI to investigate corpus callosum integrity in MS patients and age-matched controls. They also used a geometric scheme to divide the corpus callosum into five subregions on the basis of anterior-to-posterior distance, in an attempt to see whether these regions correlated with behavioral data. Regions of interest (ROIs) were drawn in the corpus callosum of each subject and were arranged as follows: one ROI (CC1) included the genu and the rostrum, three ROIs (CC2, CC3, CC4) mainly represented the body of the corpus callosum, and one ROI (CC5) covered the splenium [Bonzano et al. (2008), their Fig. 1 (http:// www.jneurosci.org/cgi/content/full/28/ $12 / 3227 / F 1)]$. The authors also used the control participants' DTI data for tractography analyses, identifying which cortical areas are connected with the various subdivisions of the corpus callosum.

MS patients demonstrated difficulty 
with bimanual coordination, presenting elevated values for $\mathrm{IHI}_{\text {onset }}$ and $\mathrm{IHI}_{\text {offset }}$ with respect to control participants; however, neither group showed a significant difference in performance at the different tapping frequencies, as can be seen in Figure 2 of Bonzano et al. (2008) (http:// www.jneurosci.org/cgi/content/full/28/ $12 / 3227 / F 2)$. Results for movement sequencing showed significantly longer touch duration for MS patients compared with control subjects; however, no difference was found for intertapping interval between groups. In agreement with other studies (Lenzi et al., 2007; Lin et al., 2007), MS patients had a significant reduction in corpus callosum FA compared with the control group for all five subregions, indicating callosal damage in the form of atrophy and/or dysfunction in those with MS. This decrease in FA values in individuals with MS may be the result of several factors including demyelination or a decrease in axonal density/integrity.

Partitioning the corpus callosum is a useful approach given that each subregion has different cortical connections. The strong correlations between behavioral performance $\left(\mathrm{IHI}_{\text {onset }}\right.$ ) and mean FA in both the CC1 and CC2 regions in MS patients [Bonzano et al. (2008), their Fig. 4 (http://www.jneurosci.org/cgi/content/ full/28/12/3227/F4)] are interesting and informative given the myelin losses associated with the disease. These correlations provide evidence that impaired bimanual coordination is related to disrupted interhemispheric communication in prefrontal and motor areas. However, correlations for the control participants were not presented. This would have added to the strength of the argument, particularly given that the authors note the heterogeneity of the corpus callosum. Although the correlations are strong and provide insight into structural and corresponding functional changes in MS, having a direct comparison with healthy controls would indicate whether a similar relationship holds, or whether such correlations are only present in the case of impairment.

The use of DTI allowed Bonzano et al. (2008) to examine in greater detail the white matter connections in their subjects. The inclusion of tractography in controls, using their five subregions of the corpus callosum as ROIs, adds to the understanding of callosal connections in healthy subjects. That being said, understanding the white matter connections in pathology, particularly MS, is an important future step. Previous work using DTI in MS patients has shown increased diffusivity and decreased FA in areas of demyelination (Nucifora et al., 2007). Moreover, tractography done on normalappearing white matter in MS patients has shown abnormal diffusion in the pyramidal tract (Lin et al., 2007). Tractography analyses in MS patients would further our understanding of changes in myelination in MS. This data would also add to the strength of Bonzano et al.'s (2008) argument that deficits in bimanual coordination in MS may be caused by impaired information exchange.

Bonzano et al. (2008) state that their results"emphasize that the effect of FA in corpus callosum on movement control is unique to bimanual coordination." However, Lenzi et al. (2007) showed reduced FA values in an area of the corpus callosum through which sensory-motor and premotor areas pass during a repetitive unimanual finger-to-thumb tapping task. Using activation patterns derived from functional magnetic resonance imaging (fMRI) along with DTI information, Lenzi et al. (2007) demonstrated an increase in activation bilaterally in primary motor, premotor, and cingulate cortices in MS patients. This suggests that in individuals with MS, there is either a reduction of inhibition to the ipsilateral hemisphere because of callosal damage, thereby facilitating recruitment of ipsilateral uncrossed motor output, or the ipsilateral activation may be the result of cortical plasticity (e.g., synaptic reorganization). Additional fMRI analysis on the functional activation differences between MS and controls would be valuable to report in conjunction with DTI data.

Previous studies on callosotomy patients have provided evidence that temporal control in bimanual tapping is not restricted to corpus callosum. It has been shown that temporal coupling during finger tapping is not affected in patients who have undergone callosotomy (Ivry and Hazeltine, 1999). In fact, event-related temporal control has been considered one of the major functions of the cerebellum (Ivry et al., 2002). The task that Bonzano et al. (2008) used contains an explicit temporal representation of the time between successive contacts between fingers. It may be that a bimanual coordination task that is more continuous (Kennerley et al., 2002) would exhibit even stronger correlations with corpus callosum FA values in MS patients. The lack of difference in intertapping interval between MS patients and controls suggests the involvement of cerebellum or other subcortical control for the discrete, repetitive action tested here. Although the authors provide plausible interpretations for their results, it may be that cerebellar white matter changes in MS also underlie impairments in bimanual coordination. This question remains open to future investigation.

\section{References}

Bonzano L, Tacchino A, Roccatagliata L, Abbruzzese G, Mancardi GL, Bove M (2008) Callosal contributions to simultaneous bimanual finger movements. J Neurosci 28:3227-3233.

Ivry RB, Hazeltine E (1999) Subcortical locus of temporal coupling in the bimanual movements of a callosotomy patient. Hum Mov Sci 18:345-375.

Ivry RB, Spencer RM, Zelaznik HN, Diedrichsen J (2002) The cerebellum and event timing. Cerebellum: recent developments in cerebellar research. Ann N Y Acad Sci 978:302-317.

Kennerley SW, Diedrichsen J, Hazeltine E, Semjen A, Ivry RB (2002) Callosotomy patients exhibit temporal uncoupling during continuous bimanual movements. Nat Neurosci 5:376-381.

Lenzi D, Conte A, Mainero C, Frasca V, Fubelli F, Totaro P, Caramia F, Inghilleri M, Pozzilli C, Pantano P (2007) Effect of corpus callosum damage on ipsilateral motor activation in patients with multiple sclerosis: a functional and anatomical study. Hum Brain Mapp 28:636-644.

Lin F, Yu C, Jiang T, Li K, Chan P (2007) Diffusion tensor tractography-based group mapping of the pyramidal tract in relapsingremitting multiple sclerosis patients. AJNR Am J Neuroradiol 28:278-282.

Nucifora PG, Verma R, Lee SK, Melhem ER (2007) Diffusion-tensor MR imaging and tractography: exploring brain microstructure and connectivity. Radiology 245:367-384.

Swinnen SP, Wenderoth N (2004) Two hands, one brain: cognitive neuroscience of bimanual skill. Trends Cogn Sci 8:18-25. 